\title{
Correlation Analysis of Breast and Thyroid Nodules: A Cross-Sectional Study
}

\author{
Jingtai Chen ${ }^{1, *}$ \\ Zhou $\mathrm{Xu}^{\mathrm{l}, *}$ \\ Lingmi Hou' \\ Yunhui Tang' \\ Shuangqiang Qian' \\ Hongyu $\mathrm{Pu}^{\mathrm{I}}$ \\ Juan Tang ${ }^{2}$ \\ Yanchun Gao' \\ 'Department of Thyroid and Breast \\ Surgery, Affiliated Hospital of North \\ Sichuan Medical College, Nanchong, \\ 637000, Sichuan, People's Republic of \\ China; ${ }^{2}$ Department of Burns and Plastic \\ Surgery, Affiliated Hospital of North \\ Sichuan Medical College, Nanchong, \\ 637000, Sichuan, People's Republic of \\ China \\ *These authors contributed equally to \\ this work
}

\begin{abstract}
Objective: Based on physical examination, to explore the relationship between breast mass $(\mathrm{BM})$ and thyroid nodule (TN) prevalence, and to further explore other related factors that affect the occurrence of BM and TN.

Methods: From January 1, 2018, to January 1, 2021, 12,538 female subjects received breast and thyroid ultrasound examinations at the same time in the health examination center of the Affiliated Hospital of North Sichuan Medical College. Univariate analysis and multivariate logistic analysis were used to screen the relevant factors affecting TN and BM, and propensity score matching was used to further verify the results of the relationship between breast and thyroid.

Results: A total of 4975 (39.7\%) of the included subjects have BM and a total of 6315 (50.4\%) have TN,2557 (20.4\%) had both BM and TN. The logistic regression results show that patients with TN are more likely to suffer from BM [OR $=1.185,95 \%$ CI (1.099-1.278), $\mathrm{p}<0.0001]$. In addition, age, free T4, HDL, height, BMI, systolic blood pressure, diastolic blood pressure, and albumin are independent factors affecting the occurrence of BM; patients with $\mathrm{BM}$ are more likely to have TN $[\mathrm{OR}=1.180,95 \%$ CI $(1.094-1.272), \mathrm{p}<0.0001]$, and age, free T3, free T4, AST, ALT, albumin, height, and BMI are independent influencing factors on the occurrence of TN. The result of propensity score matching confirmed the relationship between $\mathrm{BM}$ and $\mathrm{TN}$.

Conclusion: There is a bidirectional pathogenic relationship between BM and TN, women with $\mathrm{BM}$ are at increased risk of $\mathrm{TN}$, and women with $\mathrm{TN}$ are more likely to have BM. Thyroid hormone is not only related to the occurrence of TN but also affects the occurrence of BM.
\end{abstract}

Keywords: breast, thyroid, nodule, propensity score analysis

\section{Introduction}

Breast and thyroid diseases are becoming more and more common in the population and significantly impact women's health. ${ }^{1,2}$ The discovery of nodules is often the first sign that attracts people's attention in the early stages of breast or thyroid disease. Previous studies have shown that some factors, such as age, thyroid function, fasting glucose, sex, and blood lipid, may affect the risk of TN and BM. ${ }^{3-7}$

Ultrasound is a commonly used imaging method for breast and thyroid diseases ${ }^{8,9}$ and therefore plays an important role in health screening. Previous prospective and retrospective studies ${ }^{10-14}$ have shown an interaction between thyroid and breast diseases, but most of them ${ }^{11,13,14}$ have focused on the relationship between thyroid and breast cancers, and neglected the early signs of malignant diseases. In particular, the interrelationship between breast disease and thyroid hormones is still controversial at
Correspondence: Yanchun Gao Department of Thyroid and Breast Surgery, Affiliated Hospital of North Sichuan Medical College, Nanchong, Sichuan, People's Republic of China Email boyangIIIII@I63.com 
this stage. ${ }^{15}$ One of the essential objectives of this study was to investigate the relationship between thyroid hormone levels and the prevalence of BM in patients through a crosssectional study with a large sample.

Therefore, this study aimed to explore the prevalence of $\mathrm{TN}$ and $\mathrm{BM}$ in women and related factors by collecting thyroid ultrasound and breast ultrasound examination data from health checkups. This study will help to analyze the prevalence trend of $\mathrm{TN}$ and BMand contribute to early screening and prevention of breast and thyroid diseases.

\section{Materials and Methods}

\section{Basic Information}

A total of 12,538 females who received breast and thyroid ultrasound examinations in the health examination center of Affiliated Hospital of North Sichuan Medical College from January 2018 to January 2021 were selected.

\section{Inclusive Criteria}

1) All women who received comprehensive health examination (including thyroid, breast ultrasound examinations, fasting blood glucose, liver function, thyroid function and blood lipid test at least) from January 2018 to January 2021. 2) Women aged 18 or above.

\section{Exclusion Criteria}

1) Patients under 18 years old; 2) Male patients; 3) Patients with missing relevant data (such as age, thyroid function, thyroid or breast ultrasound report missing).

The design and purpose of this study are in line with the ethical principles of medical research in the Helsinki Declaration and approved by the Ethics Committee of the Affiliated Hospital of North Sichuan Medical College.

\section{Ultrasound Image Acquisition and}

\section{Analyses}

\section{Ultrasound Assessment of Thyroid}

Using HI VISION Preirus (Hitachi Aloka Medical, Ltd.), L9 linear array probe, frequency $5 \sim 13 \mathrm{mhz}$, with RET function and tissue diffuse quantitative analysis function, the patient took the pillow supine position, head back, fully exposed the neck examination area, routine examination of thyroid, thyroid nodules, calcification in nodules, bilateral thyroid lobe size and isthmus thickness, etc. Thyroid was classified according to Kwak Thyroid Imaging Reporting and Data System (TI-RADS) standards. ${ }^{16}$

\section{Ultrasound Assessment of Breast}

PHILIPS IU22 color Doppler ultrasound system/Mindray DC-80 ultrasound system, L9-3 broadband line array probe, frequency 7.5-10 MHz, mechanical index 0.07 . SonoVue (SV; Bracco, spa, Milan, Italy) was used as the contrast agent. Patients lie supine, fully expose the chest, and the high-frequency L9-3 broadband linear array probe was applied to scan the bilateral breast and axillary separately. Ultrasound was applied to check the size, number, and echo of the lesion, and color Doppler was used to observing the blood flow in the lesion. The image was analyzed and diagnosed by two experienced doctors. Breast lesions were evaluated by Breast Imaging Reporting and Data System (BI-RADS) standards. ${ }^{17} \mathrm{BM}$ is defined as breast lesions with three-dimensional characteristics of shape, edge and direction under ultrasound, including breast cyst, mixed or solid mass and intraductal mass, ${ }^{18}$ and breast adenopathy for which BI-RADS classification was performed.

\section{Blood Sample Analysis \\ Blood Routine Test}

Mindray BC-6800 hematology analyzer was used;

\section{Liver Function Test}

The liver function was measured by Roche cobas c701 analyzer.

\section{Blood Lipid Detection}

The instrument used is Hitachi 7600 automatic biochemical analyzer, and the reagent is the matching reagent of the machine; enzyme coupling method was used for the detection of triglyceride (TG) and total cholesterol (TC), the direct method was used for the detection of highdensity lipoprotein cholesterol (HDL), and low-density lipoprotein cholesterol (LDL) Selective clearance method was used for detection, and very-low-density lipoprotein cholesterol (VLDL) was calculated.

\section{Thyroid Function Assay}

All samples were analyzed using Siemens ADVIA Centaur. The reference intervals of FT4 was $0.89-1.76$ ng/dl, FT3 was $2.3-4.2 \mathrm{pg} / \mathrm{mL}$, TSH was $0.55-4.78 \mathrm{IU} / \mathrm{mL}$.

\section{Glucose Determination}

Glucose oxidase method and Hitachi 7170S automated analyzer were used for glucose detection. 


\section{Test Method}

Peripheral venous blood was collected from all blood samples at least 8 hours after fasting and analyzed in the laboratory of Affiliated Hospital of North Sichuan Medical College. All operations are carried out by our professional staff in strict accordance with the instrument or reagent operation instructions.

\section{Statistical Analysis}

Stata 15 and SPSS 23 were used for statistical analysis, with $\mathrm{P}<0.05$ as the difference was statistically significant. Kolmogorov-Smirnov (KS) test was used to test the normality of continuous variables. The measurement data in line with normal distribution was expressed by $(\mathrm{x} \pm \mathrm{s})$, and a $t$-test was used for comparison between groups. The measurement data in non-normal distribution was expressed by Medians (p25-p75), and the Kruskal-Wallis test was used for comparison between groups. The counting data were described by $\mathrm{n}(\%)$, and a chi-square test was used to a comparison between groups.

After univariate analysis of all the factors and considering the statistical significance and medical significance of the relevant factors, the covariates were selected and then included in the logistic regression analysis. We used binary logistic regression to explore the relationship between TN, BM and other covariates. In addition, we used multivariate logistic regression to explore the relationship between TN with different TI-RADS grades and BM with different BI-RADS grades and other included covariates.

To further confirm the association of $\mathrm{TN}$ and $\mathrm{BM}$, we conducted a sensitivity analysis using propensity score matching(PSM). ${ }^{19}$ Taking TN or BM as dependent variable, age, liver function examination, blood lipid level, fasting blood glucose and thyroid function related examination results as independent variables, the propensity score was estimated by logistic regression. A 1:1 matched analysis using nearest-neighbor matching with a caliper distance of 0.02 without replacement was performed based on the estimated propensity score of each patient. Finally, paired chi-square was used to test the relationship between TN and BM.

\section{Results}

A total of 4975 (39.7\%) of the included subjects have BM, and a total of 6315 (50.4\%) have TN,2557 (20.4\%) had both BM and TN.

Univariate analysis showed statistically significant differences in age, height, BMI, diastolic blood pressure, systolic blood pressure, free $\mathrm{T} 3$, free $\mathrm{T} 4$, triglycerides, LDL, HDL, total cholesterol, glucose, albumin, AST, and ALT (Table 1), and the effect of different levels of thyroid function on thyroid and breast nodules was also statistically significant (Table 2).

Table I Anthropometric and Biochemical Characteristics of Subjects with Different BM and TN Status by Ultrasonography

\begin{tabular}{|c|c|c|c|c|c|}
\hline Variable & Negative & BM & TN & Coexistence of BM and TN & P-value \\
\hline Age (years) & $42(32-50)$ & $4 I(33-48)$ & $49(42-55)$ & $46(40-5 I)$ & $<0.0001$ \\
\hline Height $(\mathrm{cm})$ & $156(152-159)$ & $156(153-160)$ & $155(15 \mid-159)$ & $156(152-160)$ & $<0.0001$ \\
\hline BMI & $22.64(20.8 I-24.73)$ & $22.21(20.57-24.13)$ & $23.53(21.60-25.53)$ & $22.97(21.19-24.90)$ & $<0.0001$ \\
\hline SBP & $114(105-125)$ & $112(105-122)$ & $120(109-134)$ & $117(108-129)$ & $<0.0001$ \\
\hline DBP & $68(62-76.5)$ & $68(62-75)$ & $72(65-8 I)$ & 71 (64-79) & $<0.0001$ \\
\hline $\mathrm{HDL}(\mathrm{mmol} / \mathrm{L})$ & $1.50(1.29-1.73)$ & $1.50(1.30-1.74)$ & $1.46(1.25-1.70)$ & $1.48(1.26-1.73)$ & $<0.0001$ \\
\hline $\mathrm{LDL}(\mathrm{mmol} / \mathrm{L})$ & $2.63(2.14-3.16)$ & $2.59(2.14-3.10)$ & $2.81(2.29-3.33)$ & $2.68(2.20-3.21)$ & $<0.0001$ \\
\hline $\mathrm{TG}(\mathrm{mmol} / \mathrm{L})$ & $1.02(0.77-1.44)$ & $1.00(0.75-1.37)$ & $1.16(0.86-1.62)$ & $1.09(0.82-1.50)$ & $<0.0001$ \\
\hline $\mathrm{TC}(\mathrm{mmol} / \mathrm{L})$ & $4.75(4.19-5.40)$ & $4.70(4.17-5.30)$ & $4.94(4.37-5.58)$ & $4.80(4.27-5.42)$ & $<0.0001$ \\
\hline Fasting Glucose (mmol/L) & $4.82(4.53-5.15)$ & $4.82(4.52-5.12)$ & $4.91(4.59-5.29)$ & $4.84(4.53-5.17)$ & $<0.0001$ \\
\hline $\mathrm{FT} 3(\mathrm{pg} / \mathrm{mL})$ & $2.93(2.73-3.16)$ & $2.91(2.72-3.13)$ & $2.92(2.73-3.12)$ & $2.90(2.73-3.10)$ & 0.023 \\
\hline FT4(ng/dl) & $1.24(1.14-1.37)$ & $1.25(1.14-1.37)$ & $1.23(1.13-1.35)$ & $1.24(1.14-1.36)$ & 0.024 \\
\hline TSH(ulU/mL) & $2.60(1.74-3.83)$ & $2.65(1.77-3.85)$ & $2.54(1.69-3.82)$ & $2.52(1.73-3.69)$ & 0.081 \\
\hline AST & $21(18-25)$ & $20(18-24)$ & $22(19-26)$ & $21(18-25)$ & $<0.0001$ \\
\hline ALT & $14(||-20)$ & $13(10-19)$ & $15(|2-2|)$ & $14(||-20)$ & $<0.0001$ \\
\hline Total bilirubin & $14.0(\mid 1.5-17.6)$ & $14.2(|1.5-| 7.8)$ & $14.0(|| .4-17.3)$ & I4.2(|I.6-|7.5) & 0.094 \\
\hline Plasma albumin & $46.2(44.6-47.9)$ & $46.1(44.6-47.8)$ & $46.0(44.4-47.6)$ & $45.9(44.3-47.5)$ & $<0.0001$ \\
\hline
\end{tabular}

Abbreviations: BM, breast mass; TN, thyroid nodule; HDL, high-density lipoprotein; LDL, low-density lipoprotein; TG, triglycerides;TC, total cholesterol; FT3, free-T3; FT4, free-T4; TSH, thyroid-stimulating hormone; ALT, alanine aminotransferase; AST, aspartate aminotransferase; SBP, systolic blood pressure; DBP, diastolic blood pressure. 
Table 2 Effect of BM and TN by Baseline Thyroid Hormone Level

\begin{tabular}{|c|c|c|c|c|c|}
\hline Variable & $\begin{array}{l}\text { Negative } \\
(n=3805)\end{array}$ & $B M(n=24 \mid 8)$ & TN $(n=3758)$ & $\begin{array}{l}\text { Coexistence of BM and TN } \\
\qquad(n=2557)\end{array}$ & P-value \\
\hline \multicolumn{6}{|l|}{ FT3: n (\%) } \\
\hline$<2.3$ & $4 I$ (I.I\%) & 37 (1.5\%) & $23(0.6 \%)$ & $26(1.0 \%)$ & 0.005 \\
\hline $2.3-4.2$ & 3465 (91.1\%) & 2275 (94.1\%) & 3506 (93.3\%) & 2423 (94.8\%) & $<0.0001$ \\
\hline$>4.2$ & 299 (7.9\%) & 106 (4.4\%) & $229(6.1 \%)$ & 108 (4.2\%) & $<0.0001$ \\
\hline \multicolumn{6}{|l|}{ FT4: n (\%) } \\
\hline$<0.89$ & 43 (1.1\%) & 29 (1.2\%) & $28(0.7 \%)$ & $18(0.7 \%)$ & 0.099 \\
\hline $0.89-1.76$ & 3452 (90.7\%) & 2276 (94.1\%) & 3493 (92.9\%) & 2434 (95.2\%) & $<0.0001$ \\
\hline$>1.76$ & 310 (8.1\%) & II 3 (4.7\%) & 237 (6.3\%) & 105 (4.1\%) & $<0.0001$ \\
\hline \multicolumn{6}{|l|}{ TSH: n (\%) } \\
\hline$<0.55$ & 93 (2.4\%) & 55 (2.3\%) & 89 (2.4\%) & 46 (I.8\%) & 0.355 \\
\hline $0.55-4.78$ & 3156 (82.9\%) & 2029 (83.9\%) & 3124 (83.1\%) & $2 \mid 44$ (83.8\%) & 0.661 \\
\hline$>4.78$ & $556(14.6 \%)$ & 334 (I3.8\%) & 545 (14.5\%) & 367 (14.4\%) & 0.839 \\
\hline
\end{tabular}

Notes: The reference (normal) range of FT4: $0.89-1.76 \mathrm{ng} / \mathrm{dl}, \mathrm{FT3}: 2.3-4.2 \mathrm{pg} / \mathrm{mL}$, TSH: $0.55-4.78 \mathrm{IU} / \mathrm{mL}$.

According to the result of binary logistic regression, we can conclude that the occurrence risk of BM was higher in women with TN than those without TN $[\mathrm{OR}=1.185,95 \%$ CI $(1.099-1.278)]$. The differences in age, free T4, HDL, height, BMI, systolic blood pressure, diastolic blood pressure, and albumin also remained statistically significant (Table 3).

The results of binary logistic regression analysis with $\mathrm{TN}$ as the dependent variable showed that the occurrence risk of $\mathrm{TN}$ in women with $\mathrm{BM}$ was higher than that in women without $\mathrm{BM}[\mathrm{OR}=1.180,95 \%$ CI (1094-1.272), $\mathrm{p}<0.0001]$, and the difference was still statistically significant after correcting for other covariates. Age, free T3, free T4, AST, ALT, albumin, height, and BMI remained statistically significant in the multivariate logistic regression (Table 4).

After RADS grading, the results of multivariate logistics regression showed that the strongest adjusted correlation was noted in BI-RADS category $\geq 4 \mathrm{~A} \quad \mathrm{BM}$ $(\mathrm{OR}=2.011)$, which indicated that women with high malignant possible $\mathrm{TN}$ had a higher probability of suffering from malignant BM. (Table 5)

The strongest adjusted correlation was noted in TIRADS category $\geq 4 \mathrm{~A}$ TN $(\mathrm{OR}=1.338)$, higher than the OR between TN and BM of other grades (1.231 and 1.194, respectively), again indicating a closer relationship
Table 3 Multivariable-Adjusted Odds Ratios for BM

\begin{tabular}{|c|c|c|c|c|c|c|}
\hline Variable & B & S.E. & Wald & P-value & $\begin{array}{l}\text { Odds } \\
\text { Ratio }\end{array}$ & $95 \% \mathrm{Cl}$ \\
\hline Age & -0.11 & 0.002 & 23.429 & $<0.0001$ & 0.989 & $0.985-0.993$ \\
\hline Height & 0.019 & 0.004 & 28.259 & $<0.0001$ & 1.019 & $1.012-1.026$ \\
\hline BMI & -0.025 & 0.007 & 11.099 & 0.001 & 0.976 & $0.962-0.990$ \\
\hline SBP & -0.006 & 0.002 & 7.495 & 0.006 & 0.994 & $0.990-0.998$ \\
\hline DBP & 0.008 & 0.003 & 6.487 & 0.011 & 1.008 & $1.002-1.013$ \\
\hline \multicolumn{7}{|l|}{$\mathrm{FT3}$} \\
\hline$<2.3$ & 0.25 & 0.182 & 1.890 & 0.169 & 1.283 & $0.899-1.832$ \\
\hline $2.3-4.2$ & \multicolumn{6}{|c|}{ I (reference) } \\
\hline$>4.2$ & -0.055 & 0.259 & 0.045 & 0.832 & 0.947 & $0.570-1.573$ \\
\hline \multicolumn{7}{|l|}{ FT4 } \\
\hline$<0.89$ & -0.028 & 0.201 & 0.019 & 0.891 & 0.973 & $0.656-1.442$ \\
\hline $0.89-1.76$ & \multicolumn{6}{|c|}{ I (reference) } \\
\hline$>1.76$ & -0.571 & 0.256 & 4.958 & 0.026 & 0.565 & $0.342-0.934$ \\
\hline TG & 0.034 & 0.030 & 1.274 & 0.259 & 1.035 & $0.975-1.098$ \\
\hline HDL & 0.191 & 0.085 & 5.089 & 0.024 & 1.211 & $1.025-1.429$ \\
\hline LDL & 0.060 & 0.061 & 0.972 & 0.324 & 1.062 & $0.942-1.197$ \\
\hline TC & -0.081 & 0.062 & 1.681 & 0.195 & 0.922 & $0.816-1.042$ \\
\hline Fasting glucose & -0.043 & 0.022 & 3.776 & 0.052 & 0.958 & $0.917-1.000$ \\
\hline AST & -0.005 & 0.003 & 2.483 & 0.115 & 0.995 & $0.988-1.001$ \\
\hline ALT & 0.003 & 0.002 & 1.291 & 0.256 & 1.003 & $0.998-1.007$ \\
\hline $\begin{array}{l}\text { Plasma } \\
\text { albumin }\end{array}$ & -0.027 & 0.008 & 11.051 & 0.001 & 0.974 & $0.958-0.989$ \\
\hline TN & 0.170 & 0.039 & 19.390 & $<0.0001$ & 1.185 & $1.099-1.278$ \\
\hline
\end{tabular}


Table 4 Multivariable-Adjusted Odds Ratios for TN

\begin{tabular}{|c|c|c|c|c|c|c|}
\hline Variable & B & S.E. & Wald & P-value & Odds Ratio & $95 \% \mathrm{Cl}$ \\
\hline Age & 0.052 & 0.002 & 491.225 & $<0.0001$ & 1.053 & $1.049-1.058$ \\
\hline Height & 0.025 & 0.004 & 46.220 & $<0.0001$ & 1.025 & $1.018-1.032$ \\
\hline BMI & 0.027 & 0.007 & 13.602 & $<0.0001$ & 1.028 & $1.013-1.043$ \\
\hline SBP & 0.004 & 0.002 & 3.828 & 0.050 & 1.004 & $1.000-1.008$ \\
\hline DBP & 0.003 & 0.003 & 0.705 & 0.401 & 1.003 & $0.997-1.008$ \\
\hline \multicolumn{7}{|l|}{ FT3 } \\
\hline$<2.3$ & -0.645 & 0.194 & 11.123 & 0.001 & 0.524 & $0.359-0.766$ \\
\hline $2.3-4.2$ & \multicolumn{6}{|c|}{ I (reference) } \\
\hline$>4.2$ & 0.346 & 0.257 & $1.81 \mathrm{I}$ & 0.178 & 1.413 & $0.854-2.337$ \\
\hline \multicolumn{7}{|l|}{ FT4 } \\
\hline$<0.89$ & -0.362 & 0.208 & 3.018 & 0.082 & 0.697 & $0.463-1.047$ \\
\hline $0.89-1.76$ & \multicolumn{6}{|c|}{ I (reference) } \\
\hline$>1.76$ & -0.500 & 0.253 & 3.914 & 0.048 & 0.606 & $0.369-0.995$ \\
\hline \multicolumn{7}{|l|}{ TSH } \\
\hline$<0.55$ & -0.180 & 0.129 & 1.935 & 0.164 & 0.835 & $0.648-1.076$ \\
\hline $0.55-4.78$ & \multicolumn{6}{|c|}{ I (reference) } \\
\hline$>4.78$ & -0.016 & 0.054 & 0.081 & 0.776 & 0.985 & $0.885-1.095$ \\
\hline TG & 0.048 & 0.031 & 2.285 & 0.131 & 1.049 & $0.986-1.115$ \\
\hline $\mathrm{HDL}$ & -0.022 & 0.085 & 0.067 & 0.796 & 0.978 & $0.827-1.156$ \\
\hline LDL & 0.007 & 0.061 & 0.012 & 0.913 & 0.993 & $0.88 I-1.120$ \\
\hline $\mathrm{TC}$ & -0.069 & 0.063 & 1.211 & 0.271 & 0.933 & $0.826-1.055$ \\
\hline Fasting Glucose & -0.008 & 0.021 & 0.140 & 0.708 & 0.992 & $0.952-1.034$ \\
\hline AST & -0.011 & 0.004 & 8.863 & 0.003 & 0.989 & $0.982-0.996$ \\
\hline ALT & 0.005 & 0.002 & 4.694 & 0.030 & 1.005 & $1.000-1.010$ \\
\hline Plasma albumin & -0.016 & 0.008 & 3.954 & 0.047 & 0.984 & $0.968-1.000$ \\
\hline BM & 0.165 & 0.038 & 18.557 & $<0.0001$ & 1.180 & $1.094-1.272$ \\
\hline
\end{tabular}

between TN and BM of higher malignant potential. (Table 6).

In addition, we pooled the incidence of $\mathrm{TN}$ and $\mathrm{BM}$ according to the TI/BI-RADS classification of breast and thyroid (Table 7).

After propensity score matching, 4664 pairs were successfully matched between TN group and non-TN groups, and 2014 women in the TN group suffered from BM. There were 1815 patients with BM in the non-TN group, and the paired chi-square results showed that patients with thyroid nodules were more likely to have breast nodules ( $\mathrm{P}$ $<0.0001) .4966$ pairs of BM-group and non-BM groups were successfully matched, 2556 patients in BM group and 2358 patients in the non-BM group had $\mathrm{TN}$, and the paired chi-square results showed that patients with BM were more likely to have $\mathrm{TN}(\mathrm{P}<0.0001)$. The result of PSM is the same as that of logistic regression, which proves that the results of this study are stable and reliable. Table 8 showed the characteristics before and after Propensity Score Matching.

\section{Discussion}

According to our findings, BM and $\mathrm{TN}$ usually occur synchronously, which is consistent with the results of previous studies. Shi et $\mathrm{al}^{20}$ found that the prevalence of $\mathrm{TN}$ in the normal population was $34.49 \%$, while the prevalence of thyroid nodules in the group with benign breast disease was $43.64 \%$ and in the group with breast cancer was $56.17 \%$, both significantly higher than the normal population group. Van Fossen et $\mathrm{al}^{21}$ found a 0.67 -fold 
Table 5 Multivariable-Adjusted Odds Ratios $(95 \% \mathrm{Cl})$ for BM with Different BI-RADS Categories

\begin{tabular}{|c|c|c|c|}
\hline Variable & BI-RADS 2 & BI-RADS 3 & BI-RADS $\geq 4 A$ \\
\hline TI-RADS I & \multicolumn{3}{|c|}{ I (reference) } \\
\hline TI-RADS 2 & I.I65(0.974-I.393)* & $1.028(0.894-1.183)$ & $1.156(0.825-1.62 \mid)$ \\
\hline TI-RADS 3 & $1.226(1.077-1.397)^{*}$ & I.19I(1.079-1.315)* & I.I77(0.92I-I.504) \\
\hline TI-RADS $\geq 4 \mathrm{~A}$ & $1.218(0.944-1.572)^{*}$ & $1.348(1.116-1.627)^{*}$ & $2.011(1.367-2.959) *$ \\
\hline Age & $0.987(0.980-0.994)^{*}$ & $0.988(0.983-0.993)^{*}$ & $1.007(0.994-1.020)$ \\
\hline Height & $1.017(1.006-1.028)$ & $1.021(1.013-1.030)$ & I.0II(0.99I-I.032) \\
\hline BMI & $1.004(0.983-1.027)$ & $0.958(0.942-0.975)$ & $1.005(0.965-1.046)$ \\
\hline SBP & $0.995(0.989-1.001)$ & $0.996(0.99 I-1.00 I)$ & $0.978(0.966-0.989)$ \\
\hline DBP & $1.006(0.997-1.015)$ & $1.005(0.999-1.012)$ & $1.035(1.017-1.052)$ \\
\hline \multicolumn{4}{|l|}{ FT3 } \\
\hline$<2.3$ & $1.776(1.098-2.870)$ & $1.100(0.716-1.690)$ & $0.876(0.266-2.882)$ \\
\hline $2.3-4.2$ & \multicolumn{3}{|c|}{ I (reference) } \\
\hline$>4.2$ & $0.640(0.303-1.351)$ & $1.178(0.634-2.191)$ & $1.06 \mid(0.212-5.319)$ \\
\hline \multicolumn{4}{|l|}{ FT4 } \\
\hline$<0.89$ & $0.932(0.519-1.676)$ & $1.086(0.690-1.711)$ & $0.257(0.034-1.935)$ \\
\hline $0.89-1.76$ & \multicolumn{3}{|c|}{ I (reference) } \\
\hline$>1.76$ & $0.989(0.478-2.047)$ & $0.422(0.227-0.785) *$ & $0.423(0.084-2.120)$ \\
\hline Plasma albumin & $0.972(0.949-0.996)^{*}$ & $0.978(0.960-0.996)^{*}$ & $0.956(0.914-1.000)$ \\
\hline TG & $1.029(0.939-1.127)$ & $1.032(0.96 \mathrm{I}-\mathrm{I} .108)$ & $1.066(0.928-1.224)$ \\
\hline HDL & $1.210(0.936-1.565)$ & $1.233(1.014-1.499)^{*}$ & $1.064(0.675-1.676)$ \\
\hline LDL & $1.072(0.89 \mid-1.290)$ & $1.067(0.925-1.231)$ & $0.976(0.708-1.346)$ \\
\hline TC & $0.916(0.758-1.106)$ & $0.914(0.790-1.057)$ & I.047(0.756-|.45।) \\
\hline Fasting Glucose & $1.023(0.965-1.085)$ & $0.924(0.872-0.978)^{*}$ & $0.889(0.77|-| .025)$ \\
\hline AST & $1.002(0.992-1.012)$ & $0.988(0.979-0.996)^{*}$ & $1.010(0.995-1.024)$ \\
\hline ALT & $0.996(0.989-1.004)$ & $1.007(1.002-1.013)$ & $0.994(0.983-1.006)$ \\
\hline
\end{tabular}

Note: $* p<0.05$.

increased risk of subsequent breast cancer in women with thyroid cancer and a 2-fold increased risk of subsequent thyroid cancer in women with breast cancer. In conclusion, an increasing number of researchers have found correlation between thyroid disease and breast disease, especially in terms of malignancy.

This suggests that there may be some common etiology between $\mathrm{TN}$ and $\mathrm{BM}$, which may result from genetic, environmental and hormonal factors. Current studies provide some clues to explain this relationship. Some studies have found ${ }^{22,23}$ that this may be related to mutations in PTEN, a tumor suppressor gene that predisposes women to breast, thyroid, kidney, and endometrial cancers. Similarities also exist between thyroid and breast tissues, where both thyroid follicular cells and breast cells store iodine through sodium-iodine transporter-mediated iodine uptake. $^{24}$ This suggests that iodine uptake and oxidation may play an important role in the development of mammary nodules. It has also been shown ${ }^{25}$ that $\mathrm{T} 3$ induces mammary cell proliferation by activating the same estradiol-related signaling pathway that controls cell cycle progression. Since both glands are regulated by the hypothalamic-pituitary axis, when there are abnormal thyroid hormone or estrogen levels, this may lead to the simultaneous occurrence of TN and BM in a person.

Our results showed that $39.7 \%$ of the included 12,538 women had BM, 50.4\% had $\mathrm{TN}$, and $20.4 \%$ had both BM and TN. The nodule incidence was higher compared to the results of previous studies, which may be related to the following:1) Only women were included in this study, and women have a higher prevalence of $\mathrm{BM}$ and $\mathrm{TN}$ relative to men; ${ }^{26,27}$ 2) The subjects included in this study were from medical checkup centers, and generally patients are more likely 
Table 6 Multivariable-Adjusted Odds Ratios $(95 \% \mathrm{Cl})$ for TN with Different TI-RADS Categories

\begin{tabular}{|c|c|c|c|}
\hline Variable & TI-RADS 2 & TI-RADS 3 & TI-RADS $\geq 4 A$ \\
\hline BI-RADS I & \multicolumn{3}{|c|}{ I (reference) } \\
\hline BI-RADS 2 & $1.145(0.958-1.370)^{*}$ & $1.23 \mathrm{I}(\mathrm{I} .08 \mathrm{I}-\mathrm{I} .40 \mathrm{I})^{*}$ & $1.212(0.939-1.564)$ \\
\hline BI-RADS 3 & $1.018(0.885-1.170)$ & $1.194(1.081-1.318)^{*}$ & $1.338(1.108-1.617)^{*}$ \\
\hline $\mathrm{BI}-\mathrm{RADS} \geq 4 \mathrm{~A}$ & $1.136(0.810-1.592)$ & $1.187(0.93|-| .5 \mid 4)$ & $2.020(1.373-2.97 I) *$ \\
\hline Age & $1.030(1.023-1.037)^{*}$ & $1.063(1.057-1.068)^{*}$ & $1.055(1.044-1.065)^{*}$ \\
\hline Height & 1.021 (1.010-1.033) & $1.024(1.016-1.032)$ & $1.039(1.023-1.055)$ \\
\hline BMI & 1.021 (0.998-1.044) & 1.031 (1.014-1.048) & $1.027(0.996-1.059)$ \\
\hline SBP & $1.002(0.995-1.008)$ & $1.003(0.999-1.008)$ & $1.010(1.002-1.019)$ \\
\hline DBP & $1.003(0.994-1.012)$ & $1.002(0.996-1.009)$ & $1.004(0.992-1.016)$ \\
\hline \multicolumn{4}{|l|}{ FT3 } \\
\hline$<2.3$ & $0.789(0.442-1.407)$ & $0.389(0.243-0.622)^{*}$ & $0.922(0.429-1.985)$ \\
\hline $2.3-4.2$ & \multicolumn{3}{|c|}{ I (reference) } \\
\hline$>4.2$ & $2.426(0.927-6.349)$ & $1.312(0.757-2.276)$ & $1.070(0.352-3.254)$ \\
\hline \multicolumn{4}{|l|}{ FT4 } \\
\hline$<0.89$ & $0.394(0.174-0.890)^{*}$ & $0.865(0.552-1.354)$ & $0.530(0.184-1.528)$ \\
\hline $0.89-1.76$ & \multicolumn{3}{|c|}{ I (reference) } \\
\hline$>1.76$ & $0.343(0.131-0.896) *$ & $0.673(0.391-1.156)$ & $0.694(0.233-2.070)$ \\
\hline \multicolumn{4}{|l|}{ TSH } \\
\hline$<0.55$ & $0.358(0.199-0.645)^{*}$ & $0.987(0.75|-| .298)$ & $0.935(0.55 I-I .586)$ \\
\hline $0.55-4.78$ & \multicolumn{3}{|c|}{ I (reference) } \\
\hline$>4.78$ & $1.043(0.886-1.229)$ & $0.982(0.872-1.107)$ & $0.862(0.678-1.096)$ \\
\hline Plasma albumin & $1.010(0.985-1.036)$ & $0.973(0.956-0.991)^{*}$ & $0.987(0.953-1.022)$ \\
\hline TG & $1.042(0.95 I-|| 4 \mid)$. & $1.053(0.984-1.127)^{*}$ & $1.049(0.913-1.206)$ \\
\hline HDL & $\mathrm{I} .040(0.806-|.34|)$ & $0.955(0.79|-I| 53)$. & $1.011(0.685-1.494)$ \\
\hline LDL & $0.914(0.765-1.092)$ & $0.996(0.872-1.139)$ & $1.244(0.936-1.655)$ \\
\hline $\mathrm{TC}$ & I.047 (0.87I-I.258) & $0.926(0.807-1.062)^{*}$ & $0.725(0.542-0.971) *$ \\
\hline Fasting Glucose & $1.027(0.968-1.090)$ & $0.978(0.934-1.024)$ & $0.994(0.9 \mid 4-1.080)$ \\
\hline AST & $0.995(0.984-1.006)$ & $0.988(0.980-0.996)^{*}$ & $0.987(0.972-1.002)$ \\
\hline ALT & $1.001(0.993-1.009)$ & $1.006(1.001-1.011)^{*}$ & $1.009(1.000-1.019)$ \\
\hline
\end{tabular}

Note: $* P<0.05$.

Table 7 Ultrasound BI-/TI-RADS Categories of Breast and Thyroid

\begin{tabular}{|l|l|l|}
\hline Variable & Breast $(\mathbf{n = 1 2 , 5 3 8 )}$ & Thyroid $(\mathbf{n = 1 2 , 5 3 8 )}$ \\
\hline RADS I & $7563(60.3 \%)$ & $6223(49.6 \%)$ \\
RADS 2 & $1499(11.9 \%)$ & $1467(11.7 \%)$ \\
RADS 3 & $3099(24.7 \%)$ & $4154(33.1 \%)$ \\
RADS $\geq$ 4A & $377(3.0 \%)$ & $694(5.5 \%)$ \\
\hline
\end{tabular}

Abbreviation: BI-/TI-RADS, Breast/Thyroid Imaging Reporting and Data System. to go to hospitals for comprehensive health checkups after unintentionally finding out they have $\mathrm{BM}$ or $\mathrm{TN} ; 3$ ) Consistent with the current epidemiological trend of thyroid and breast cancers, the incidence of $\mathrm{TN}$ and $\mathrm{BM}$ is increasing year by year; ${ }^{28,29}$ 4) Compared to previous studies, the definition of BM is not exactly the same; for example, we explicitly defined breast adenopathy with BI-RADS grading as BM. 


\begin{tabular}{|c|c|c|c|c|c|c|c|c|c|c|c|}
\hline \multirow{3}{*}{ 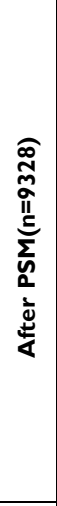 } & 0 & $\overline{\bar{o}}$ & $\frac{\hat{\lambda}}{\hat{o}}$ & $\frac{\stackrel{\mathrm{T}}{0}}{0}$ & $\bar{\circ}$ & $\frac{1}{0}$ & 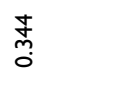 & $\stackrel{\circ}{\pi}$ & 今̊ & స్ & $\overline{\bar{o}}$ \\
\hline & $\mid \begin{array}{l}0 \\
0 \\
0 \\
0 \\
\mathbf{z} \\
\mathbf{1} \\
\dot{1} \\
\mathbf{0} \\
\mathbf{z}\end{array}$ & \& & $\stackrel{\substack{\widehat{\sigma} \\
\stackrel{n}{1}}}{\stackrel{n}{=}}$ & 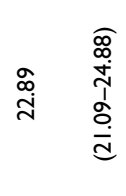 & $\stackrel{\substack{\mathfrak{I} \\
=}}{\stackrel{1}{0}}$ & $\curvearrowright \quad \begin{array}{l}\hat{\alpha} \\
1 \\
\hat{\sigma}\end{array}$ & 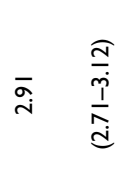 & 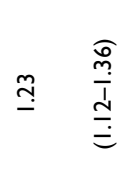 & 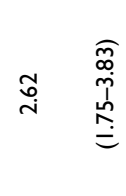 & 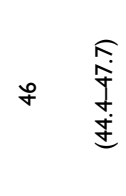 & $\stackrel{\circ}{\circ}$ \\
\hline & $\begin{array}{l}0 \\
0 \\
\vdots \\
\mathbf{z} \\
\mathbf{Z}\end{array}$ & 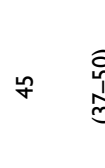 & $\stackrel{\substack{\text { r } \\
\stackrel{n}{n}}}{=}$ & 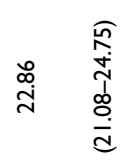 & 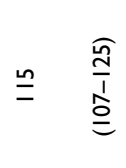 & $\begin{array}{l}\text { E } \\
\hat{N} \\
\text { d }\end{array}$ & 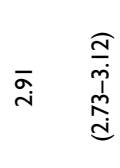 & 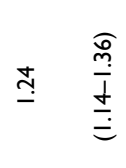 & $\begin{array}{l}\widehat{\alpha} \\
\hat{j} \\
0 \\
\stackrel{j}{=}\end{array}$ & 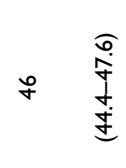 & 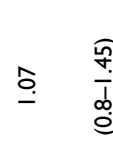 \\
\hline \multirow{3}{*}{ 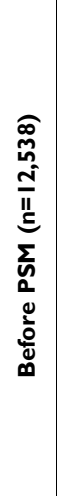 } & 0 & 호 & के & 홍 & 호 & 호 & ö. & চ̊̀. & \%̊. & 호. & 흥 \\
\hline & \begin{tabular}{|l|}
0 \\
$\vdots$ \\
$\vdots$ \\
$\vdots$ \\
$\mathbf{z}$ \\
$\mathbf{z}$ \\
$\dot{1}$ \\
$\mathbf{z}$ \\
$\mathbf{z}$
\end{tabular} & 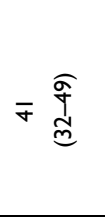 & 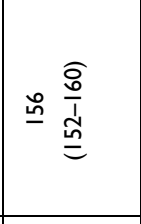 & 交 & 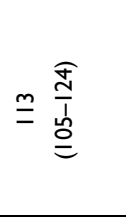 & $\infty \underset{0}{\infty}$ & 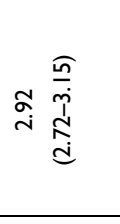 & 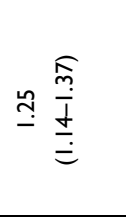 & 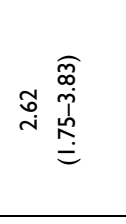 & 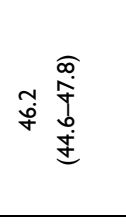 & 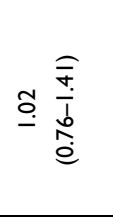 \\
\hline & $\begin{array}{l}0 \\
\overline{0} \\
0 \\
0 \\
\mathbf{z} \\
1\end{array}$ & 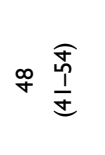 & 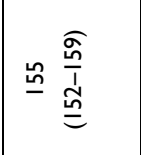 & 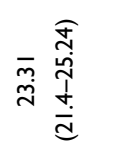 & $\stackrel{\infty}{=} \underset{\frac{m}{d}}{\frac{\hat{\sigma}}{\delta}}$ & $\approx \stackrel{\widehat{o}}{a}$ & 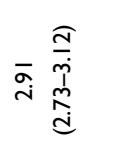 & 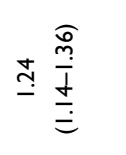 & 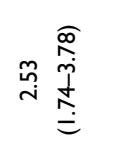 & 子 & 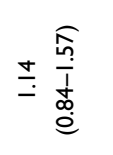 \\
\hline \multirow{3}{*}{ 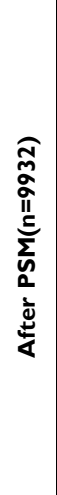 } & $a$ & 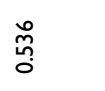 & 角 & 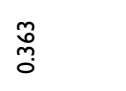 & $\stackrel{\tilde{m}}{0}$ & 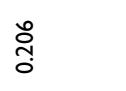 & $\stackrel{0}{0}$ & 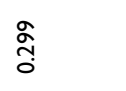 & $\underset{\frac{m}{i}}{\hat{0}}$ & $\overline{\substack{\tilde{O} \\
O}}$ & $\begin{array}{l}\infty \\
\stackrel{\infty}{\circ} \\
0\end{array}$ \\
\hline & 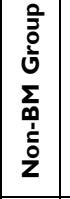 & F $\underset{\hat{m}}{\widehat{m}}$ & 号 & 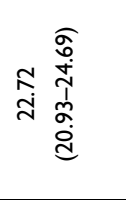 & $\stackrel{\substack{\widehat{N} \\
=}}{\stackrel{\underline{b}}{=}}$ & 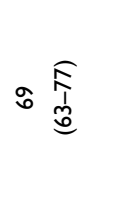 & 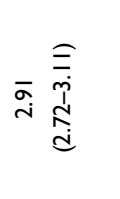 & 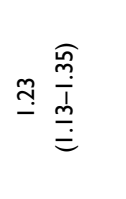 & 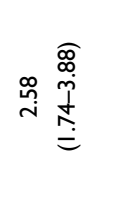 & 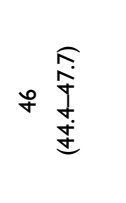 & 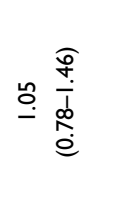 \\
\hline & $\begin{array}{l}0 \\
\vdots \\
\vdots \\
\Sigma \\
\Sigma\end{array}$ & 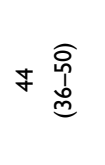 & 号 & 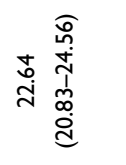 & 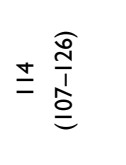 & 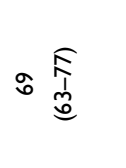 & $\bar{i} \underset{i}{\stackrel{\widehat{c}}{\stackrel{d}{d}}}$ & 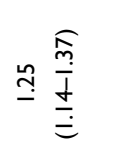 & 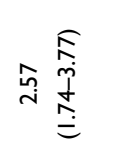 & 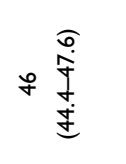 & 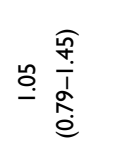 \\
\hline \multirow{3}{*}{ 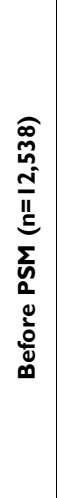 } & 2 & 형 & क्ष & 형 & 형 & চо & চ̊̀ & $\begin{array}{l}\overline{0} \\
\text { o. }\end{array}$ & $\begin{array}{l}\stackrel{n}{\circ} \\
0 \\
0\end{array}$ & $\frac{\overline{0}}{0}$ & 형 \\
\hline & 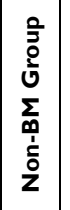 & \& & 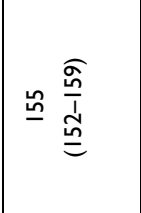 & 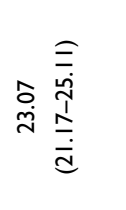 & 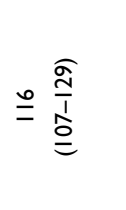 & 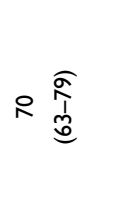 & 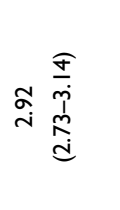 & 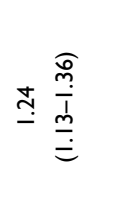 & 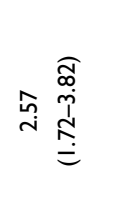 & 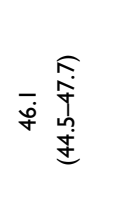 & $=\frac{\frac{\widehat{\hat{n}}}{\frac{1}{\infty}}}{\stackrel{\frac{1}{\infty}}{\stackrel{\infty}{\infty}}}$ \\
\hline & $\begin{array}{l}0 \\
\vdots \\
0 \\
\vdots \\
\Sigma \\
\Sigma \\
\infty\end{array}$ & ఫ & $\stackrel{\widehat{o}}{\stackrel{\frac{\sigma}{d}}{N}}$ & 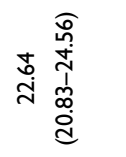 & $\pm \frac{\stackrel{o}{\frac{1}{1}}}{=}$ & 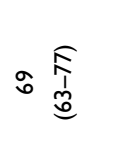 & 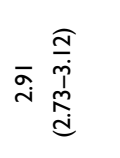 & 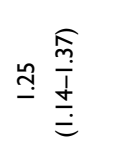 & 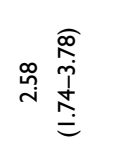 & 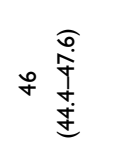 & 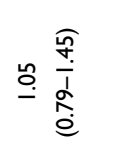 \\
\hline 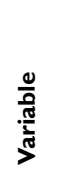 & & 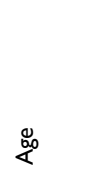 & 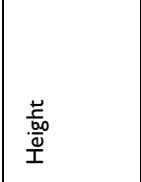 & $\overline{\sum_{\infty}}$ & 总 & $\begin{array}{l}\text { 商 } \\
\end{array}$ & $\stackrel{m}{\mathscr{H}}$ & 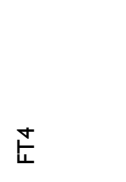 & $\begin{array}{c}\frac{I}{\varphi} \\
\qquad\end{array}$ & 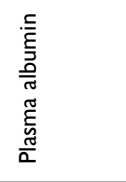 & $\stackrel{\cup}{\vdash}$ \\
\hline
\end{tabular}




\begin{tabular}{|c|c|c|c|c|c|c|}
\hline ণิ ָิ & $\frac{\circ}{0}$ & $\frac{\swarrow}{\circ}$ & 莽 & 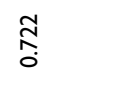 & $\begin{array}{l}\hat{\infty} \\
\text { ổ } \\
0\end{array}$ & $\begin{array}{l}\text { 苫 } \\
\text { O. }\end{array}$ \\
\hline 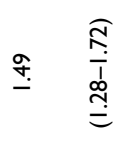 & 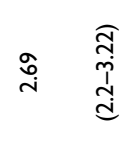 & 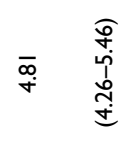 & 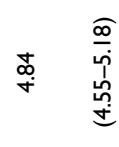 & 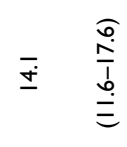 & $\begin{array}{l}\stackrel{\widehat{T}}{\infty} \\
\stackrel{\infty}{=}\end{array}$ & $\stackrel{\overline{\bar{I}}}{\Xi}$ \\
\hline 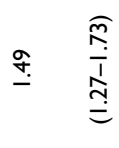 & 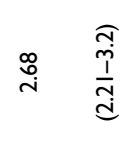 & 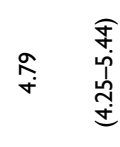 & 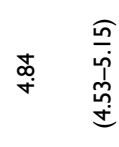 & 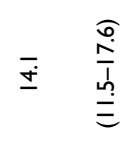 & $\begin{array}{l}\widehat{\widehat{N}} \\
\stackrel{\infty}{=}\end{array}$ & $\stackrel{\text { Iิ }}{\stackrel{1}{\Xi}}$ \\
\hline 형 & 호. & $\bar{o}$ & চ̊̀. & $\stackrel{\infty}{\circ}$ & ֻัّ & $\frac{t}{0}$ \\
\hline 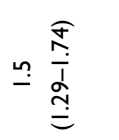 & 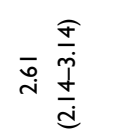 & 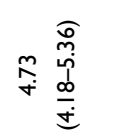 & 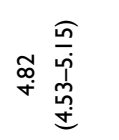 & 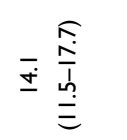 & $\bar{\sim} \underset{\stackrel{\widehat{N}}{\underline{\Phi}}}{\underline{\Phi}}$ & 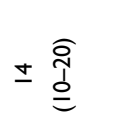 \\
\hline 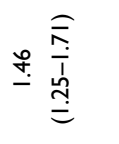 & 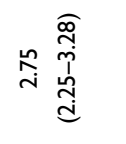 & 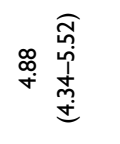 & 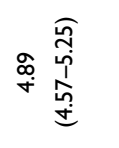 & 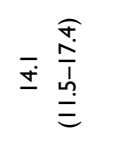 & $\bar{\sim} \stackrel{\widehat{\hat{W}}}{=}$ & 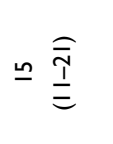 \\
\hline స్ & 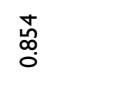 & $\bar{\alpha}$ & 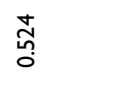 & $\begin{array}{l}\text { 20 } \\
\text { مُ }\end{array}$ & $\stackrel{\hat{\lambda}}{\hat{0}}$ & $\begin{array}{l}\bar{\alpha} \\
\infty \\
0\end{array}$ \\
\hline 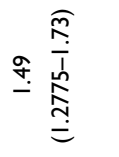 & 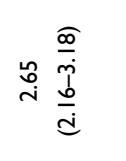 & 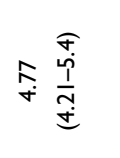 & 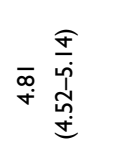 & 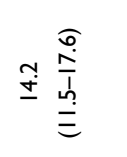 & 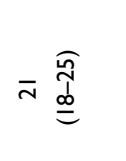 & 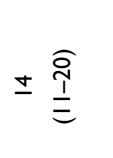 \\
\hline 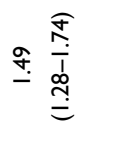 & 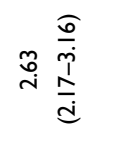 & 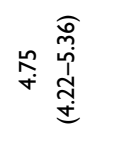 & 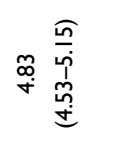 & 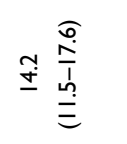 & 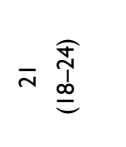 & 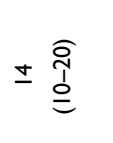 \\
\hline 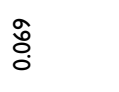 & 흥 & চ্ণ & চे & 흥 & $\bar{o}$ & $\stackrel{1}{0}$ \\
\hline 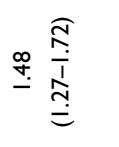 & 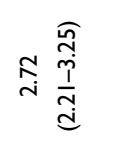 & 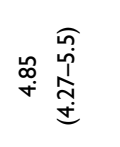 & 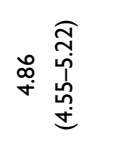 & 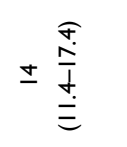 & $\bar{\sim} \underset{\stackrel{\widehat{N}}{\underline{\infty}}}{=}$ & $\underline{\underline{\underline{I}}} \underline{\widehat{\bar{I}}}$ \\
\hline 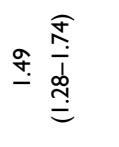 & 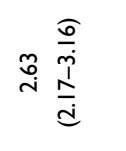 & 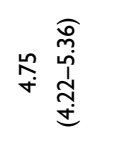 & 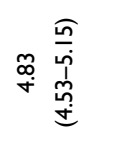 & 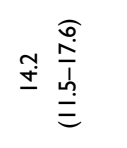 & 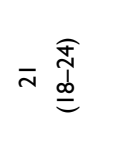 & 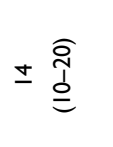 \\
\hline 뭄 & $\vec{a}$ & $\stackrel{U}{\vdash}$ & 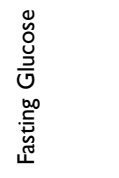 & 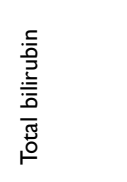 & 歺 & $\stackrel{5}{\gtrless}$ \\
\hline
\end{tabular}


In our study, age was found to be an independent risk factor for the occurrence of $\mathrm{TN}$, and this result is consistent with the results of previous studies. ${ }^{30}$ The results in multivariate logistic regression indicated that age was a protective factor for BI-RADS grade 2 and $3 \mathrm{BM}$, which may be associated with the high incidence of benign breast hyperplasia in young women. As with the results of previous studies, ${ }^{31,32}$ this paper also found that abnormal lipid levels can influence the occurrence of TN. In this paper, ALT and AST were also found to be associated with the occurrence of TN. Recently, Komori et $\mathrm{al}^{33}$ found an epidemiological correlation between head and neck tumors and hepatitis $\mathrm{B}$ virus infection. So it may be that abnormal liver function due to hepatitis virus infection causes a statistical correlation between $\mathrm{TN}$ occurrence and liver function, and hepatitis $\mathrm{B}$ virus infection is a serious public health problem in China, where tens of millions of hepatitis B virus carriers. ${ }^{34,35}$ The association between hepatitis $B$ virus infection and $\mathrm{TN}$ requires our attention, and further studies are necessary to investigate the underlying mechanisms causing this phenomenon.

A large number of studies have investigated the relationship between thyroid hormone levels and breast carcinogenesis, and the role of thyroid hormones on the development of breast cancer has received widespread attention. On the one hand, Rasool et $\mathrm{al}^{36}$ found significantly higher T3 and T4 levels in the breast cancer group than the normal population, suggesting that high levels of T 3 and T 4 are significantly associated with the risk of breast cancer. A 30-year cohort study conducted by Sogaard et $\mathrm{al}^{37}$ in Denmark also showed that women with hyperthyroidism were at greater risk of breast cancer. Women with hypothyroidism had a slightly lower risk of breast cancer. A previous in vitro experiment similarly confirmed that thyroid hormones promote nuclear estrogen receptor alpha $(\mathrm{ER} \alpha)$-dependent cells and promote angiogenesis and breast cancer cell growth through activation of ER $\alpha$-mediated pathways. $^{25}$ However, in a study by Kuijpens et al, ${ }^{38}$ thyroid hypofunction and lower free T4 levels were associated with an increased risk of breast cancer in postmenopausal women. These studies suggest that we may have an imbalance between estrogen and thyroid hormones promoting the development and progression of breast cancer. Our study found that higher free T4 reduced the risk of developing BM. This relationship was mainly observed in BI-RADS 3 breast, as retrospective studies are susceptible to confounding by other confounding factors, especially estrogen, which, together with T4, acts on ER $\alpha$ to promote breast cell proliferation. We also do not know whether any of the women examined were taking oral levothyroxine for hypothyroidism, among other factors, which may confound our conclusions. However, previous studies and our findings suggest an association between thyroid hormone levels and the development of breast disease, and more researches are required to clarify the specific mechanisms of action between thyroid function and BM.

This paper contains a large sample size and multiple covariates, as did the previous study, ${ }^{6}$ but an important advantage of this study is the introduction of thyroid hormones that are extremely important for the occurrence of $\mathrm{TN}$ and BM, which makes us be able to explain the effect of thyroid hormone on thyroid and breast. Moreover, this article uses propensity score matching to verify the relationship between $\mathrm{TN}$ and $\mathrm{BM}$, making our results more reliable. There are some shortcomings in this study: certain important covariates were not included, especially estrogen. Many previous studies ${ }^{39-41}$ have shown that thyroxine and estradiol share a signaling pathway that regulates the growth of breast cancer cells. Because women rarely undergo sex hormone screening during health checkups, the effect of other hypothalamicpituitary axis-related hormones on $\mathrm{TN}$ or BM development was not discussed in this article to ensure sample size. Further studies are needed to assess whether sex hormones affect the development of thyroid and breast disease.

In conclusion, elucidating the common pathogenesis between $\mathrm{BM}$ and $\mathrm{TN}$ will have important implications for the diagnosis and prevention of these diseases. Especially in light of current studies, both BM and TN have a high prevalence, and there is an association between the pathogenesis of both. Therefore, clinicians may consider routine imaging of the thyroid or breast at the time of physical examination, especially if a high TI-RADS-graded TN or a high BI-RADS-graded BM is found, or if other risk factors are combined, necessitating examination of the other gland. A large sample, prospective, multi-center study is needed further to explore the pathological relationship between thyroid and breast nodules.

\section{Ethics Approval and Consent to Participate}

The Ethics Committee approved this study of the Affiliated Hospital of North Sichuan Medical College. Because of the retrospective nature of the study, patient 
informed consent for inclusion was waived. All information was anonymous. The study was conducted in accordance with the Declaration of Helsinki.

\section{Acknowledgments}

We are particularly grateful to all the people who have given us help with our article.

\section{Disclosure}

The authors declare that they have no conflicts of interest in this work.

\section{References}

1. Wiltshire JJ, Drake TM, Uttley L, Balasubramanian SP. Systematic review of trends in the incidence rates of thyroid cancer. Thyroid. 2016;26(11):1541-1552. doi:10.1089/thy.2016.0100

2. Krishnamoorthy Y, Rajaa S, Giriyappa DK, Bharathi A, Velmurugan B, Ganesh K. Worldwide trends in breast cancer incidence from 1993 to 2012: age-period-cohort analysis and joinpoint regression. $J$ Res Med Sci. 2020;25(1):98. doi:10.4103/jrms. JRMS_708_19

3. Pace R, Rahme E, Dasgupta K. Gestational diabetes mellitus and risk of incident primary cancer: a population-based retrospective cohor study. J Diabetes. 2020;12(1):87-90. doi:10.1111/1753-0407.12988

4. Kim EY, Chang Y, Lee KH, et al. Serum concentration of thyroid hormones in abnormal and euthyroid ranges and breast cancer risk: a cohor study. Int J Cancer. 2019;145(12):3257-3266. doi:10.1002/ijc.32283

5. Lipscombe LL, Fischer HD, Austin PC, et al. The association between diabetes and breast cancer stage at diagnosis: a population-based study. Breast Cancer Res Treat. 2015;150 (3):613-620. doi:10.1007/s10549-015-3323-5

6. Li H, Wang Z, Liu JS, et al. Association between breast and thyroid lesions: a Cross-Sectional Study based on ultrasonography screening in China. Thyroid. 2020;30(8):1150-1158. doi:10.1089 thy. 2019.0184

7. Anil C, Akkurt A, Ayturk S, Kut A, Gursoy A. Impaired glucose metabolism is a risk factor for increased thyroid volume and nodule prevalence in a mild-to-moderate iodine deficient area. Metabolism. 2013;62(7):970-975. doi:10.1016/j.metabol.2013.01.009

8. Niell BL, Freer PE, Weinfurtner RJ, Arleo EK, Drukteinis JS. Screening for breast cancer. Radiol Clin North Am. 2017;55 (6):1145-1162. doi:10.1016/j.rcl.2017.06.004

9. Liu R, Zhang B. Role of ultrasound in the management of thyroid nodules and thyroid cancer. Zhongguo Yi Хие Кe Xие Yuan Xие Bao. 2017;39(3):445-450. doi:10.3881/j.issn.1000-503X.2017.03.025

10. Dobrinja C, Scomersi S, Giudici F, et al. Association between benign thyroid disease and breast cancer: a single center experience. Bmc Endocr Disord. 2019;19(1):104. doi:10.1186/s12902-019-0426-8

11. Bolf EL, Sprague BL, Carr FE. A linkage between thyroid and breast cancer: a common etiology? Cancer Epidemiol Biomarkers Prev. 2019;28(4):643-649. doi:10.1158/1055-9965.EPI-18-0877

12. Joseph KR, Edirimanne S, Eslick GD. The association between breast cancer and thyroid cancer: a meta-analysis. Breast Cancer Res Treat. 2015;152(1):173-181. doi:10.1007/s10549-015-3456-6

13. An JH, Hwangbo Y, Ahn HY, et al. A possible association between thyroid cancer and breast cancer. Thyroid. 2015;25(12):1330-1338. doi:10.1089/thy.2014.0561

14. Arer IM, Yabanoglu H, Kus M, Akdur A, Avci T. Retrospective analysis of patients with synchronous primary breast and thyroid carcinoma. Eur J Breast Health. 2018;14(2):80-84. doi:10.5152/ ejbh.2018.3853
15. Cooter M, Soliman AS, Pavlou P, et al. Incidence and time trends of cancer in cyprus over 11 years (1998--2008). Tumori. 2015;101 (1):8-15. doi:10.5301/tj.5000204

16. Kwak JY, Han KH, Yoon JH, et al. Thyroid imaging reporting and data system for US features of nodules: a step in establishing better stratification of cancer risk. Radiology. 2011;260(3):892-899. doi:10.1148/radiol.11110206

17. Magny SJ, Shikhman R, Keppke AL Breast imaging reporting and data system. 2021.

18. Margolies LR, Pandey G, Horowitz ER, Mendelson DS. Breast Imaging in the era of big data: structured reporting and data mining. Am J Roentgenol. 2016;206(2):259-264. doi:10.2214/ AJR.15.15396

19. Baser O. Too much ado about propensity score models? Comparing methods of propensity score matching. Value Health. 2006;9 (6):377-385. doi:10.1111/j.1524-4733.2006.00130.x

20. Shi Y, Li X, Ran L, et al. Study on the status of thyroid function and thyroid nodules in chinese breast cancer patients. Oncotarget. 2017;8 (46):80820-80825. doi:10.18632/oncotarget.20542

21. Van Fossen VL, Wilhelm SM, Eaton JL, Mchenry CR. Association of thyroid, breast and renal cell cancer: a population-based study of the prevalence of second malignancies. Ann Surg Oncol. 2013;20 (4):1341-1347. doi:10.1245/s10434-012-2718-3

22. Ngeow J, Stanuch K, Mester JL, Barnholtz-Sloan JS, Eng C. Second malignant neoplasms in patients with cowden syndrome with underlying germline PTEN mutations. $J$ Clin Oncol. 2014;32 (17):1818-1824. doi:10.1200/JCO.2013.53.6656

23. Ngeow J, Sesock K, Eng C. Breast cancer risk and clinical implications for germline PTEN mutation carriers. Breast Cancer Res Treat. 2017;165(1):1-8. doi:10.1007/s10549-015-3665-z

24. Zbigniew S. Role of iodine in metabolism. Recent Pat Endocr Metab Immune Drug Discov. 2017;10(2):123-126. doi:10.2174/ 1872214811666170119110618

25. Hall LC, Salazar EP, Kane SR, Liu N. Effects of thyroid hormones on human breast cancer cell proliferation. J Steroid Biochem Mol Biol. 2008;109(1-2):57-66. doi:10.1016/j.jsbmb.2007.12.008

26. Liu X, Sun J, Fang W, Xu Y, Zhu Z, Liu Y. Current iodine nutrition status and morbidity of thyroid nodules in Mainland China in the past 20 years. Biol Trace Elem Res. 2021. doi:10.1007/s12011-020-02565-2

27. Swamy N, Rohilla M, Raichandani S, Bryant-Smith G. Epidemiology of male breast diseases: a 10-year institutional review. Clin Imaging. 2021;72:142-150. doi:10.1016/j.clinimag.2020.11.027

28. Chen W, Zheng R, Baade PD, et al. Cancer statistics in China, 2015. CA Cancer J Clin. 2016;66(2):115-132. doi:10.3322/caac.21338

29. Sharen G, Zhang B, Zhao R, Sun J, Gai X, Lou H. Retrospective epidemiological study of thyroid nodules by ultrasound in asymptomatic subjects. Chin Med J. 2014;127(9):1661-1665.

30. Tian $\mathrm{C}, \mathrm{Bu} \mathrm{Y}, \mathrm{Ji}$ C, et al. Iodine nutrition and the prevalence status of thyroid nodules in the population: a cross-sectional survey in Heilongjiang Province, China. Biol Trace Elem Res. 2020. doi:10.1007/s12011-020-02442-y

31. Buscemi S, Massenti FM, Vasto S, et al. Association of obesity and diabetes with thyroid nodules. Endocrine. 2018;60(2):339-347. doi:10.1007/s12020-017-1394-2

32. Liu J, Wang C, Tang X, et al. Correlation analysis of metabolic syndrome and its components with thyroid nodules. Diabetes Metab Syndr Obes. 2019;12:1617-1623. doi:10.2147/DMSO.S219019

33. Komori MF, Kimura T, Kariya S, et al. Epidemiological correlations between head and neck cancer and hepatitis B core antibody positivity. Anticancer Res. 2020;40(4):2393-2403. doi:10.21873/ anticanres. 14209

34. Su QD, Zhang S, Wang F, et al. Epidemiological distribution of hepatitis B virus genotypes in 1-29-year-olds in the mainland of China. Vaccine. 2020;38(51):8238-8246. doi:10.1016/j.vaccine.20 20.09.083 
35. Zu J, Li M, Zhuang G, et al. Estimating the impact of test-and-treat strategies on hepatitis B virus infection in China by using an age-structured mathematical model. Medicine. 2018;97(16):e0484. doi:10.1097/MD.0000000000010484

36. Rasool M, Naseer MI, Zaigham K, et al. Comparative Study of alterations in Tri-iodothyronine (T3) and Thyroxine (T4) hormone levels in breast and ovarian cancer. Pak J Med Sci. 2014;30 (6):1356-1360. doi:10.12669/pjms.306.5294

37. Sogaard M, Farkas DK, Ehrenstein V, Jorgensen JO, Dekkers OM, Sorensen HT. Hypothyroidism and hyperthyroidism and breast cancer risk: a nationwide cohort study. Eur J Endocrinol. 2016;174 (4):409-414. doi:10.1530/EJE-15-0989

38. Kuijpens JL, Nyklictek I, Louwman MW, Weetman TA, Pop VJ, Coebergh JW. Hypothyroidism might be related to breast cancer in post-menopausal women. Thyroid. 2005;15(11):1253-1259. doi:10.1089/thy.2005.15.1253
39. Milosevic Z, Tanic N, Bankovic J, et al. Genetic alterations in quadruple malignancies of a patient with multiple sclerosis: their role in malignancy development and response to therapy. Int J Clin Exp Pathol. 2014;7(4):1826-1833.

40. Sun LM, Lin CL, Liang JA, Kao CH. Authors' reply to radiotherapy did not increase thyroid cancer risk among women with breast cancer: a nationwide population-based cohort study. Int $J$ Cancer. 2016;138(7):1803-1804. doi:10.1002/ijc.29906

41. Dinda S, Sanchez A, Moudgil V. Estrogen-like effects of thyroid hormone on the regulation of tumor suppressor proteins, p53 and retinoblastoma, in breast cancer cells. Oncogene. 2002;21 (5):761-768. doi:10.1038/sj.onc. 1205136

\section{Publish your work in this journal}

The International Journal of General Medicine is an international, peer-reviewed open-access journal that focuses on general and internal medicine, pathogenesis, epidemiology, diagnosis, monitoring and treatment protocols. The journal is characterized by the rapid reporting of reviews, original research and clinical studies across all disease areas. The manuscript management system is completely online and includes a very quick and fair peer-review system, which is all easy to use. Visit http://www.dovepress.com/ testimonials.php to read real quotes from published authors. 